\title{
Predictores de la conducta antisocial juvenil: un modelo ecológico ${ }^{1}$
}

\author{
Martha Frías-Armenta, Amelia Eréndida López-Escobar, Sylvia Guadalupe Díaz-Méndez \\ Universidad de Sonora, México
}

\begin{abstract}
Resumen
Este estudio pone a prueba un modelo ecológico como marco teórico explicativo de la antisocialidad juvenil. 204 jóvenes mexicanos que cursaban la educación secundaria o preparatoria contestaron un cuestionario con preguntas acerca de la violencia intrafamiliar, su conducta antisocial, la ingesta de alcohol de sus madres, los problemas de conducta escolar, algunas características del ambiente familiar, escolar y del barrio y las actitudes acerca de la violencia. Los datos fueron analizados a través de un modelo estructural en el cual las variables investigadas constituyeron factores e índices que representaban a los niveles de la teoría ecológica. Los resultados mostraron que el microsistema tuvo un efecto directo en la conducta antisocial de los menores, el exosistema mostró un efecto también directo en el microsistema y por lo tanto uno indirecto en la conducta antisocial de los menores, y el macrosistema tuvo un efecto directo en el exosistema y uno indirecto en la antisocialidad de los jóvenes. Lo anterior parece respaldar el modelo ecológico, como explicación coherente de la conducta antisocial en los menores.
\end{abstract}

Palabras clave: conducta antisocial, jóvenes, modelo ecológico, ecuaciones estructurales.

\begin{abstract}
The aim of this research was to test an ecological model as explanation of juvenile delinquency. 204 Mexican students of junior and high school answered a questionnaire containing questions about family violence, antisocial behavior, child abuse, mother and father alcohol consumption, as well as some family, school, and neighborhood environmental characteristics. Observed variables constituted factors and indexes that represented the ecological theory. Structural equation modeling was used to analyze data. Results showed that the microsystem had a direct effect on antisocial behavior of youths, the exosystem had also a direct effect on the microsystem and an indirect effect on antisocial behavior, while the macrosystem had a direct effect on the exosystem and an indirect effect on antisocial behavior. These results seem to support the ecological model as an appropriate explanation of juvenile antisocial behavior.
\end{abstract}

Key words: antisocial behavior, juveniles, ecological model, structural equations.

L a delincuencia juvenil y las conductas antisociales en menores son problemas complejos que atraen la atención de la sociedad por sus manifestaciones cada vez más frecuentes y violentas. En México fueron arrestados 58,720 menores en el año de 1997 y 56,448 en 1998 (Instituto Nacional de Estadística, Geografía e Informática, INEGI, 1998). Estos indicadores representan únicamente los de los menores que fueron internados en los centros de atención por algún delito grave, pero el número de menores denunciados por alguna infracción es mucho mayor que el de los internados (únicamente entre el $10 \%$ al $15 \%$ son internados). Por ejemplo en la ciudad de Hermosillo, en el estado norteño de Sonora, de 300 denuncias que se recibieron en la policía en el mes de enero del 2000, únicamente 50 fueron turnadas al Consejo Tutelar para Menores. De éstos únicamente 31 fueron detenidos; el resto de los menores fue amonestado o fueron entregados en custodia familiar. Esto demuestra que los datos del INEGI no constituyen una aproximación real a la magnitud del problema, ya que la institución procesa únicamente la información proveniente de los menores que fueron arrestados o detenidos en algún Consejo o tribunal.

De acuerdo con las estadísticas del gobierno, en el estado de Sonora en 1996 se arrestaron 14, 355 jóvenes entre los 11 y los 18 años, quienes fueron consignados en los tribunales para menores. Estos representan el 6\% de la población total de jóvenes en esa entidad (Gobierno del Estado de Sonora, 1998). En el año de 1997 se atendieron 13,833 menores en las delegaciones y/o representaciones del Consejo Tutelar para Menores (Gobierno del Estado de Sonora, 1998). En cuanto a la atención externa, se dio servicio a 22,491 menores en 1997 
(Gobierno del Estado de Sonora, 1998). En total fueron atendidos 36,321 menores, los que conforman el 15\% de la población total de éstos. Los datos no consideran a los menores que fueron denunciados $\mathrm{y}$ entregados a sus padres después de recibir una amonestación, quienes constituyen la mayor parte de los jóvenes infractores. Lo anterior mostraría que un gran porcentaje de la población juvenil (quizá entre el $15 \%$ y el 30\%) en México se involucra en actividades criminales y esto constituye una preocupación que requiere la comprensión de los determinantes de la antisocialidad juvenil.

\section{Teorías explicativas}

Diversas teorías se han cuestionado el origen de la violencia en los humanos. Los filósofos y los investigadores debaten acerca de la naturaleza "noble” o "salvaje” de las personas (Freud, 1930; Lorenz, 1966). Freud y otros psicoanalistas aseguran que la agresión es el producto de un primitivo y poderoso deseo por la muerte al cual ellos llaman Tánatos. Lorenz (1966) argumenta que la violencia es instintiva. Sin embargo, los estudios científicos actuales reportan que las causas de la violencia son múltiples y éstas incluyen factores biológicos, fisiológicos, conductuales, sociales, económicos y políticos (American Psychological Association's Comission on Youth Violence, 1993; National Research Council, 1993; Ollendick, 1996).

Lerner y Galambos (1998) plantean que, de manera consistente con las condiciones individuales y las contextuales, existen factores centrales básicos en la génesis y en el desarrollo de las conductas de riesgo de la criminalidad, sobre las cuales se puede trabajar en prevención. Estos comprenden tres factores individuales y tres contextuales: 1) la edad (la iniciación temprana en una conducta de riesgo en la adolescencia); 2) las expectativas respecto de la escuela y de las calificaciones escolares; 3) conducta(s) general(es), tales como acciones inapropiadas; 4) observar influencias antisociales, ya que muchas veces más allá de los factores individuales los factores contextuales son los que conducen a la generación de conductas de riesgo; 5) las influencias de los padres, particularmente sus estilos autoritarios o permisivos; y 6) las influencias del vecindario.

\section{El modelo ecológico}

Bronfenbrenner (1987) propone una perspectiva ecológica del desarrollo de la conducta humana. Esta perspectiva concibe al ambiente ecológico como un conjunto de estructuras seriadas y estructuradas en diferentes niveles, en donde cada uno de esos niveles contiene al otro. Bronfenbrenner denomina a esos niveles el microsistema, el mesosistema, el exosistema y el macrosistema. El microsistema constituye el nivel más inmediato en el que se desarrolla el individuo (usualmente la familia); el mesosistema comprende las interrelaciones de dos o más entornos en los que la persona en desarrollo participa activamente; al exosistema lo integran contextos más amplios que no incluyen a la persona como sujeto activo; finalmente, al macrosistema lo configuran la cultura y la subcultura en la que se desenvuelve la persona y todos los individuos de su sociedad. Bronfenbrenner (1987) argumenta que la capacidad de formación de un sistema depende de la existencia de las interconexiones sociales entre ese sistema y otros. Todos los niveles del modelo ecológico propuesto dependen unos de otros y, por lo tanto, se requiere de una participación conjunta de los diferentes contextos y de una comunicación entre ellos.

Bronfenbrenner y Ceci (1994) han modificado su teoría original y plantean una nueva concepción del desarrollo humano en su teoría bio-ecológica. Dentro de esta teoría, el desarrollo es concebido como un fenómeno de continuidad y cambio de las características bio-psicológicas de los seres humanos, tanto de los grupos como de los individuos. El elemento crítico de este modelo es la experiencia que incluye no sólo las propiedades objetivas sino también las que son subjetivamente experimentadas por las personas que viven en ese ambiente. Bronfenbrenner y Ceci (1994) argumentan que, en el transcurso de la vida, el desarrollo toma lugar a través de procesos cada vez más complejos en un activo organismo bio-psicológico. Por lo tanto el desarrollo es un proceso que deriva de las características de las personas (incluyendo las genéticas) y del ambiente, tanto el inmediato como el remoto y dentro de una continuidad de cambios que ocurren en éste a través del tiempo. El modelo teórico es referido como un modelo Proceso-Persona-Contexto-Tiempo (PPCT).

Belsky (1980) retomó el modelo original de Bronfenbrenner y lo aplicó al abuso infantil. En la aplicación de Belsky, la familia representaba al microsistema; y el autor argumentaba que en este nivel más interno del modelo se localiza el entorno más inmediato y reducido al que tiene acceso el individuo. El microsistema refiere las relaciones más próximas de la persona y la familia, es el escenario que conforma este contexto inmediato. Éste puede funcionar como un contexto efectivo y positivo de desarrollo humano o puede desempeñar un papel destructivo o disruptor de este desarrollo (Bronfenbrenner, 1987). El mundo de trabajo, el vecindario, las relaciones sociales informales y los servicios constiturían al exosistema, y los valores culturales y los sistemas de creencias se incorporarían en el macrosistema. Como lo mencionábamos, para Belsky (1980) el exosistema es el segundo nivel y está compuesto por la comunidad más próxima después del grupo familiar. Ésta incluye las instituciones mediadoras entre los niveles de la cultura y el individual: la escuela, la iglesia, los medios de comunicación, las instituciones recreativas y los organismos de seguridad. La escuela constituye un lugar preponderante en el ambiente de los jóvenes; ellos permanecen una gran parte de su tiempo en este lugar, el que contribuye a su desarrollo intelectual, emocional y social. El macrosistema comprende el ambiente ecológico que abarca mucho más allá de la situación inmediata que afecta a la persona. Es el contexto más amplio y remite a las formas de organización social, los sistemas de creencias y los estilos de vida que prevalecen en una cultura o subcultura (Belsky, 1980; Bronfenbrenner, 1987). En este nivel se considera que la persona se ve afectada profundamente por hechos en los que la persona ni siquiera está presente. La integración 
en la sociedad es parte de la aculturación de los individuos a las instituciones convencionales, las normas y las costumbres (Angenent \& Man, 1996).

Emery y Laumann-Billings (1998) utilizaron el modelo ecológico para analizar las causas y las consecuencias de las relaciones familiares abusivas y establecieron a la familia como el contexto más inmediato. El contexto ecológico más amplio lo constituyeron las cualidades de la comunidad en las que está inmersa la familia, tales como la pobreza, la ausencia de servicios, la violencia, la desorganización social, la carencia de identidad dentro de sus miembros, y la falta de cohesión en ella. Por su parte, el contexto sociocultural estuvo formado por los valores y las creencias culturales.

En el presente estudio retomamos el modelo ecológico aplicado por Belsky (1980) al abuso infantil. En este modelo, Belsky propone los mismos sistemas que Bronfenbrenner, pero los define de manera diferente. Segun Belsky (1980) las relaciones dentro de la familia constituyen el vínculo más próximo y el sistema más inmediato en el que se desenvuelven los niños, al que denomina microsistema. El barrio y la escuela son contextos importantes para los menores, pero constituyen otro nivel de interacción, al cual llama el exosistema y por último, Belsky considera que la cultura constituye el macrosistema.

\section{Determinantes de la antisocialidad y de la delincuencia juvenil}

En Criminología, la inestabilidad, la desintegración y la disrupción familiar constituyen una parte central en la explicación del desarrollo de la delincuencia (Wells \& Rankin, 1991). Los conflictos familiares, la pérdida de los padres y la falta de habilidades de crianza son los factores que pudieran intervenir en el desarrollo de la antisocialidad y de la delincuencia (Morrison \& Cherlin, 1995; Widom \& Ames, 1994). McCord (2001) plantea que los padres o cuidadores forjan criminales a través de las prácticas de crianza en tres formas: 1) por la trasmisión de valores a través de sus propias acciones y las acciones que ellos aprueban, 2) en el desarrollo, por la falta de ligas con y entre los miembros de la familia, y 3) estableciendo la legitimidad de las acciones antisociales, a través de los métodos que ellos utilizan para lograr sus deseos en sus hijos/as. Los niños/as aprenden lo que es valorado dentro del ambiente familiar, sea esto bueno o malo para el resto de la sociedad (McCord, 1996).

Juby y Farrington (2001), en un estudio para identificar la relación entre la desintegración familiar y la delincuencia, encontraron que la delincuencia de los jóvenes se correlacionaba con la desintegración y el conflicto familiar, concluyendo que es el conflicto que antecede a los divorcios y no la separación de los padres lo que pudiera llevar a los jóvenes a delinquir. La violencia en el hogar, tanto el maltrato dirigido hacia los niños como el maltrato hacia las madres de éstos, resulta en un empobrecimiento del ambiente familiar. Dicho empobrecimiento causa que los niños presenten problemas en su desarrollo, manifestándose en la escuela cuando éstos ingresan a ella. Por otro lado, la violencia puede tener consecuencias devastadoras en los menores, las que pueden manifestarse como conducta antisocial o autodestructiva en los niños (Straus, 1991). Los niños que son castigados físicamente están en más riesgo de mostrar conducta antisocial (Straus, Sugarman \& Giles-Sims, 1997). Así mismo, se ha encontrado en la familia disfuncional una de las bases para el desarrollo de la delincuencia (Wells \& Rankin, 1991; Juby \& Farrington, 2001).

La escuela es el lugar en donde los jóvenes adquieren conocimientos, pero también es el escenario en donde se entrenan para las relaciones sociales y en donde se exponen a las variadas normas sociales, reglas y costumbres de su comunidad (Angenent \& Man, 1996). La forma en la que la escuela ejerce influencia en los estudiantes es a través de sus políticas, las cuales se ven reflejadas en el establecimiento de reglas y las maneras con las que se hacen cumplir las mismas. Existe una relación entre los ambientes escolares y la delincuencia; un ambiente escolar positivo permite relaciones prosociales entre estudiantes y profesores, y entre los estudiantes (Angenent \& Man, 1996; Vazsonyi \& Flannery, 1997). Es probable que en la escuela ocurra también un patrón de aprendizaje de acciones antisociales y delictivos y que algunos estímulos del contexto escolar promuevan más que otros la aparición y el mantenimiento de esas acciones negativas. El ambiente escolar es uno de los contextos más importantes de convivencia de los adolescentes con sus compañeros y es también el escenario en el que reciben más influencia de ellos. Un ambiente escolar negativo puede conducir a los escolares a comportarse antisocialmente, como lo muestran Lotz y Lee (1999). De la misma manera, Gaustad (1992) señala evidencias de que hay más confrontación entre los alumnos en las escuelas en las que existen reglas que no son claras, o reglas arbitrarias e injustas. Lo mismo ocurre cuando las conductas desviadas de los menores son ignoradas y cuando las escuelas carecen de recursos suficientes para la enseñanza.

Los vecindarios son parte de la vida diaria de los menores (Bursik, 2001). Abrahamson (1996) encontró que los vecindarios jugaban un rol importante en la asimilación de los grupos a las instituciones sociales. Ese mismo autor reporta que los menores que viven en barrios violentos manifiestan más conducta antisocial o agresiva. La delincuencia juvenil se agrupa en algunos sectores de las ciudades o regiones. En las áreas en donde hay delincuencia también existen otras formas de conducta desviada o antisocial y otros problemas sociales (Taylor, 1997), y en las ciudades la delincuencia se concentra en ciertas áreas (Peterson \& Krivo, 1993; Sampson, 1997).

Por otro lado, la delincuencia juvenil se encuentra en lugares en donde existe oportunidad para cometer delitos: en áreas comerciales, lugares de entretenimiento y en vecindarios socialmente problemáticos. Estos vecindarios problemáticos por lo general presentan condiciones de mucha pobreza en los hogares, poco mantenimiento de las casas, y poca gente que vive en éstas es propietaria de las mismas (Sampson, Raudenbush \& Earls, 1997). Existen espacios abiertos, casas y edificios abandonados y pocos lugares de recreo y áreas de servicio (Angenent \& Man, 1996; Sampson et al., 1997). 
Peterson, Krivo y Harris (2000), en un estudio llevado a cabo en comunidades en desventaja, encontraron que los vecindarios podían reducir la delincuencia con el desarrollo de ciertos tipos de instituciones locales (centros de recreo, escuelas, etcétera). Es probable que las condiciones de pobreza no sean directamente las que ocasionen la delincuencia, sino la carencia de servicios o de instituciones para el desarrollo de los menores.

Algunos autores proponen que los jóvenes, por su naturaleza, no están muy dispuestos a la integración social, y más bien buscan independencia y su propia identidad. Los jóvenes son críticos de la sociedad y señalan sus problemas; sin embargo, por lo general ellos siguen las reglas impuestas. La integración de los jóvenes en la sociedad depende de las normas a las que ellos personalmente se adhieren (Brook \& Newcomb, 1995; Hagan, Hefler, Classen, Boehnke \& Merkens, 1998).

Las actitudes sociales favorables a la agresión constituyen uno de los factores señalados como antecedentes de la delincuencia juvenil. Loeber y Hay (1997), en una revisión de la literatura, examinaron la relación entre actitudes favorables a la violencia, agresiones menores y crímenes violentos en adolescentes y preadolescentes, encontrando que las actitudes favorables a la violencia predecían la conducta delictiva de los menores (Loeber, Keenan \& Zhang, 1997).

El modelo ecológico del desarrollo representa un marco teórico para el estudio de la antisocialidad, debido a que comprende todos los entornos en los que se desenvuelve el menor. Estos escenarios, tal como los plantean Bronfenbrenner (1987) y Belsky (1980) pudieran contribuir al desarrollo de la conducta prosocial del individuo, si éstos son positivos. Sin embargo, un ambiente familiar adverso, un vecindario conflictivo, con venta de drogas, una escuela con condiscípulos antisociales, con un ambiente físico descuidado y una cultura de no respeto a las leyes, y la impunidad, pudieran generar conducta antisocial y delictiva en los menores.

El propósito del presente trabajo es el de modelar, mediante el auxilio de las ecuaciones estructurales, el esquema de la teoría ecológica, como marco explicativo de la génesis y mantenimiento de la conducta antisocial en jóvenes. Aunque este estudio se desarrolló con menores mexicanos, las similitudes de las condiciones sociales y culturales de la mayoría de las comunidades latinoamericanas podría permitir generalizar sus resultados a este ámbito mayor.

\section{Método}

\section{Participantes}

Se entrevistaron 204 jóvenes que cursaban la educación secundaria y preparatoria en escuelas de una población mexicana. Los sujetos estudiados presentaron una media de edad de 15 años. El promedio escolar de los estudiantes fue de 64 (en una escala del 0 al 100), con una desviación estándar de 33. La misma proporción de los sujetos correspondió al sexo masculino y al femenino (50\% y 50\% cada uno). La Tabla 1 nos muestra las principales características demográficas de la muestra.

Las escuelas se seleccionaron tomando en cuenta los parámetros del Instituto Nacional de Geografía e Informática (INEGI, 1998). El INEGI organiza el sistema estadístico nacional en áreas geográficas a tres niveles o áreas: Área Geoestadística Estatal (AGEE), Área Geoestadística Municipal (AGEM), y Área Geoestadística Básica (AGEB). Los AGEB constituyen la unidad fundamental del marco geoestadístico nacional y conforman una selección de los principales conceptos de la temática censal en la cual es plausible calcular indicadores específicos, y en general, realizar operaciones para obtener estadísticas derivadas (INEGI, 1998).

Para el estudio, se seleccionó un AGEB que fuera representativo de los tres estratos socioeconómicos (alto, medio, y bajo) de la ciudad y después se seleccionaron las escuelas que representaran ese AGEB. Una vez determinadas las escuelas se entrevistó a un grupo de cada grado; en la escuela secundaria se entrevistó a un grupo de primero, a uno de segundo, y a uno de tercero. En la escuela preparatoria se entrevistó a un grupo de primer semestre, a uno de tercero y a uno de quinto.

\section{Instrumentos}

Se aplicó una batería de pruebas consistente en 17 escalas, 9 de las cuales fueron elaboradas en los Estados Unidos,

Tabla 1

Medias de variables demográficas

\begin{tabular}{lccrrr}
\hline \multicolumn{1}{c}{ Variables } & $\mathrm{N}$ & Media & D.E. & Mínimo & Máximo \\
\hline Edad & 204 & 15 & 1.70 & 12 & 19 \\
Promedio escolar & 204 & 64.80 & 33.49 & 0 & 99 \\
Ingreso menor & 204 & 9.80 & 103.16 & 0 & 1300 \\
Edad madre & 173 & 38 & 3.35 & 30 & 41 \\
Edad padre & 204 & 41 & 4.61 & 35 & 47 \\
Ingreso familiar & 200 & $3,064.06$ & 6767.72 & 0 & 50000.00 \\
\hline
\end{tabular}


traducidas al español y piloteadas con anterioridad al presente estudio. Las otras 4 escalas fueron elaboradas expresamente para esta investigación. Las escalas se describen a continuación:

Conducta antisocial y delictiva. Está constituida por 17 items que miden el número de veces que el niño ha cometido un acto o conducta antisocial o delictiva especificada en el instrumento, durante los 12 meses anteriores a la entrevista, en donde: $0=$ nunca, $1=$ una 0 dos veces, $2=$ de tres a cinco veces, $3=$ de seis a diez veces, $4=$ de once a veinte veces y $5=$ más de veinte veces. Las acciones reflejan el número de veces que los menores reportaron haber golpeado a alguien, robado cosas, o hecho daños en propiedad ajena en el último año. En un estudio anterior se reportó un alfa de Cronbach de .75 para esta escala (Frías, Ramírez, Soto, Castell \& Corral, 2000).

Maltrato y castigo del padre y de la madre. Esta escala contiene diez ítems que fueron tomados de la Escala de Tácticas de Conflicto de Straus (1979; 1990) y fue administrada a todos los niños de la muestra. Estos reactivos miden la frecuencia de la violencia física y emocional del padre y de la madre hacia sus hijos, en una escala de cero a cinco, $0=$ nunca, $1=$ una vez, 2 = dos veces, $3=$ de tres a cinco veces, 4 = seis a diez veces, $5=$ once a veinte veces, $6=$ más de veinte veces. Straus (1979) reportó un alfa de Cronbach de 0.72 para los trece ítems utilizados.

Violencia entre los padres. Se utilizaron 12 items de la escala de Violencia Intramarital de Straus (1980). Ésta se contesta empleando valores que van de 0 a 6 , en donde $0=$ nunca ... 6=más de veinte veces. Al igual que en la escala de los niños, se obtuvo un alfa de .91 para los items utilizados.

Uso de alcohol de la madre y del padre. El uso de alcohol y drogas fue medido con preguntas obtenidas del Inventario de Diagnóstico de Alcohol para Padres (Reich \& Herjanic, 1989). Se utilizaron tres items de ese inventario, los cuales medían la frecuencia y la cantidad de alcohol que ingería la madre por semana. La frecuencia se midió en una escala de 6 puntos ( $0=$ nunca $\ldots 5=$ todos los días de la semana) y la cantidad en una escala de 5 puntos ( $0=$ =nada ... 4=más de seis bebidas en cada ocasión).

Ambiente familiar. Esta escala fue elaborada especialmente para el presente estudio y mide la percepción de los sujetos acerca del ambiente físico familiar. Los entrevistados reportaron acerca de lo frío, caliente, iluminada, insegura, ruidosa y deteriorada que percibían su casa, en una escala de 0 al 10 , en donde $0=$ nada y $10=$ demasiado. Se obtuvo una consistencia interna de la escala de alfa $=.82$.

Ambiente en la colonia. Se elaboraron nueve reactivos para medir la percepción que tenían los participantes acerca de su colonia (barrio). Los sujetos calificaron, en una escala que iba de 0 a 10, qué tan peligrosa, ruidosa, sucia, y oscura sentían su colonia. Además, los estudiantes reportaron la frecuencia de la venta de drogas en su barrio y la cantidad de vagos y personas embriagadas que veían en el mismo, así como la heterogeneidad de la composición social del barrio. El alfa que se obtuvo de la escala, para este estudio, fue de .85 .

Problemas escolares. Los problemas escolares fueron medidos en una escala elaborada por Frías, Corral, Moreno y
Rodríguez (2000). En la misma, los niños indicaron el número de veces que manifestaron una conducta inapropiada (indisciplina) en la escuela, considerando 6 puntos posibles de respuesta, en donde $0=$ nunca, $1=$ una 0 dos veces ... y $5=$ más de 20 veces.

Ambiente escolar. Esta escala fue elaborada especialmente para el presente estudio y mide la percepción que tienen los estudiantes de su ambiente escolar. Los sujetos reportaron la ocurrencia de peleas en la escuela, la venta de drogas, la presencia de vagos, la suciedad y el descuido de la escuela, en una escala del 0 al 10 , en donde $0=$ nada y $10=$ demasiado. Se obtuvo un alfa de .80 para esta escala.

Actitudes. Las actitudes acerca de la ley, la justicia, el gobierno, la venganza por propia mano, y la impunidad fueron medidas en una escala tipo likert de 5 puntos $(0=$ estás en completo desacuerdo, $1=$ ligeramente de acuerdo, $2=$ ni de acuerdo ni en desacuerdo, $3=$ ligeramente de acuerdo, y 4= completamente de acuerdo). Con sus respuestas, el joven manifestaba su grado de acuerdo con lo adecuado de comportamientos como mentir, obrar mal, no seguir reglas, actuar impunemente, y el tomar riesgos, entre otros.

\section{Procedimiento}

Los alumnos contestaron el cuestionario en el aula en donde recibían clases. Dos psicólogas clínicas estuvieron aclarando sus dudas y dando las instrucciones dentro del aula. A los alumnos se les informó que su participación era totalmente voluntaria y que podían dejar de contestar el cuestionario en cualquier momento. De la misma manera se les aseguró total confidencialidad de la información que se obtuviera.

\section{Análisis de datos}

Los datos fueron analizados en el programa estadístico SAS, de donde se obtuvieron estadísticas univariadas como las frecuencias de las variables categóricas, las medias y las desviaciones estándar de las variables continuas y las alfas de Cronbach de las escalas utilizadas. Los promedios de las escalas constituyeron índices que representaron a las variables a relacionar. Con estos índices se elaboró la matriz de correlaciones para probar el modelo propuesto. Esta prueba del modelo se logró utilizando ecuaciones estructurales, mediante el paquete EQS (Bentler, 1995). El modelo de ecuaciones estructurales contempla dos pasos importantes: el modelo de medición y el modelo estructural. El modelo de medición es un análisis factorial confirmatorio, mientras que el análisis estructural estima las relaciones entre los factores obtenidos en el modelo de medición. La ventaja de este sistema analítico es que permite medir al mismo tiempo los efectos directos e indirectos que tiene una variable latente u observada sobre otra(s) variables (Corral-Verdugo, 2002).

El modelo de medición de esta investigación consistió en la correlación (pesos factoriales) entre los factores exosistema y microsistema con sus correspondientes indicadores. Se presumió que el exosistema estaría formado por el ambiente de la colonia y el contexto escolar adverso y por un comportamiento escolar problemático. El microsistema lo 
formarían el abuso infantil materno y paterno, la violencia paterna, el abuso de alcohol por parte de la madre y el ambiente físico desfavorable. El macrosistema estaría conformado por un índice, que fue la suma de variables acerca de creencias y actitudes hacia la ley. Esto significa que el macrosistema fue tratado, en el modelo, como una variable manifiesta.

El modelo estructural consistió en la medición de los efectos (coeficientes estructurales) del factor microsistema en la conducta antisocial, del exosistema en el microsistema y del macrosistema en el exosistema. Se esperaban coeficientes altos y significativos $(p<.05)$ en todas las relaciones especificadas.

Para determinar si los datos respaldan el modelo teórico bajo prueba se establecen indicadores de bondad de ajuste. En el caso del EQS, se establece un indicador de bondad de ajuste estadístico que es la chi cuadrada $\left(\chi^{2}\right)$, y los indicadores prácticos que controlan el efecto del número de sujetos (N) sobre la significatividad de la prueba de $\chi 2$. Estos indicadores prácticos son el Indice Bentler-Bonett de Ajuste Normado (IBAN), el Índice Bentler-Bonett de Ajuste No Normado (IBANN) y el Índice de Ajuste Comparativo (IAC). Dado que estamos probando si el modelo teórico está respaldado por los datos, es decir, que el modelo propuesto no es significativamente diferente de las correlaciones entre todas las variables, entonces esperaríamos que el valor de $\chi^{2 \text {, que }}$ estima esa relación, sea bajo y no significativo, es decir, que su probabilidad asociada sea mayor a .05. Para los indicadores prácticos se requiere que su valor se acerque a 1.0 y que sea, al menos, superior a .90, como evidencia de que el modelo alcanzó bondad de ajuste (Bentler, 1995). También se consideró el indicador de la Raíz Cuadrada del Cuadrado Medio del Error de Aproximación (RMSEA, por sus siglas en inglés) cuyo valor deberá ser menor a .05, como evidencia de bondad de ajuste (Browne \& Cudeck, 1993).

\section{Resultados}

Análisis univariados y confiabilidad de las escalas. La Tabla 2 muestra los análisis de confiabilidad, medias y desviaciones estándar, así como los puntajes mínimos y máximos que produjeron las variables que constituyeron las escalas en el estudio. Puede observarse que las alfa fueron al menos de .60 para todas las escalas, lo cual es un indicador de su confiabilidad (Nunally \& Bernstein, 1994).

La Figura 1 muestra los resultados del modelo estructural. Primeramente se probó la validez de constructo de las dos variables latentes (exosistema y microsistema). Todos los pesos factoriales del exosistema con sus correspondientes variables indexadas (ambiente de la colonia, conducta escolar y ambiente escolar) fueron significativas $(p<.05)$. El índice de "ambiente de la colonia" obtuvo un peso factorial de .53 , mientras que el de "ambiente de colonia" tuvo uno de .48 y el de "ambiente escolar” mostró un peso factorial de .50. De la misma manera, el microsistema parece constituirse como un factor coherente, ya que todos los índices que lo forman produjeron valores altos y significativos para sus cargas factoriales. El abuso materno produjo un peso factorial de .44 con su factor de microsistema, el peso factorial de abuso paterno fue de .51 , el de la violencia paterna fue de .43 , el del alcohol materno fue de $.24 \mathrm{y}$ el del ambiente de la casa fue de .22. Se tomaron todos esos indicadores factoriales como indicios de validez de constructo de las dos variables latentes.

Al considerar el modelo estructural, se estimó el efecto del microsistema en la delincuencia, resultando un coeficiente estructural de .73. El efecto del exosistema en el microsistema se mostró como un coeficiente estructural de .74, mientras que la influencia del macrosistema en el exosistema se reflejó en un coeficiente de .50 . El modelo produjo una $R^{2}$ (coeficiente de determinación múltiple) de .56 en la estimación de la variable dependiente, lo que significa que el mismo explica el 56 por ciento de la variabilidad en la conducta antisocial de los jóvenes.

Los indicadores de bondad de ajuste fueron favorables. La chi cuadrada de este modelo fue de 53.39 (30 g.l., de diez variables manifiestas y dos factores) a la que se le asoció una $p=.005$ y los valores de IANN e IAC fueron mayores a .90. $(I A N N=.90, I A C=.93)$ y el resultado de RMSEA $=.04$. Esto significa que el modelo y sus interrelaciones se ajustan bien a los datos.

\section{Conclusiones}

Nuestros datos parecen demostrar que el modelo ecológico propuesto por Bronfenbrenner (1987) y adaptado por Belsky (1980) constituye una representación adecuada (evidenciada en su bondad de ajuste) de la generación y mantenimiento de la conducta antisocial en jóvenes. En este modelo, el microsistema explica directamente un 56\% de la varianza en la conducta antisocial, la cual es también afectada de manera indirecta por el exo y el macrosistema. Como se anticipaba, los contextos más amplios afectan a los más próximos en término de desarrollo de la conducta antisocial. En el modelo, el macrosistema tuvo un efecto positivo en el exosistema y éste en el microsistema, y finalmente este último afectó positivamente a la conducta antisocial. Lo anterior significa que el contexto cultural afectó la forma en la que los individuos se relacionaban entre sí dentro del contexto escolar y en el del barrio. El ambiente del barrio influyó en las relaciones familiares y éstas en la conducta antisocial del menor. De la misma manera, el efecto del macrosistema en el exosistema, significaría que la existencia de un sistema de creencias y actitudes ejerce influencia en la forma en la que la gente se comporta dentro de su contexto social. Los estilos de vida, la cultura y la subcultura, y la integración en la sociedad son partes de la aculturación de los individuos a las instituciones convencionales, las normas y las costumbres (Angenent \& Man, 1996).

El efecto del exosistema en el microsistema expresa que las relaciones familiares están permeadas por el ambiente, en este caso, de violencia o inestabilidad que se vive en el contexto más próximo que es el vecindario. Las condiciones físicas y sociales del barrio propician la violencia en el mismo. En los barrios pobres se presentan condiciones de carencia material en los hogares, las casas no reciben mantenimiento, existen pocos lugares de recreación y de servicio (como las 
Tabla 2

Medias y confiabilidad de escalas

\begin{tabular}{|c|c|c|c|c|c|c|}
\hline ESCALA y Variables & $\mathrm{N}$ & Min & Max & Media & D.E. & alfa \\
\hline ABUSO DEL PADRE & & & & & & .76 \\
\hline Papá me grita & 196 & 0 & 6 & 1.12 & 1.75 & \\
\hline Papá amenaza con pegarme & 196 & 0 & 6 & 0.76 & 1.54 & \\
\hline Papá me llama tonto & 196 & 0 & 6 & 1.07 & 1.79 & \\
\hline Papá me dice me correrá a golpes & 196 & 0 & 6 & 0.20 & 0.94 & \\
\hline Papá me pega en la cara & 196 & 0 & 6 & 0.30 & 1.14 & \\
\hline ABUSO DE LA MADRE & & & & & & .73 \\
\hline Mamá me grita & 196 & 0 & 6 & 1.40 & 1.85 & \\
\hline Mamá amenaza con pegarme & 196 & 0 & 6 & 0.75 & 1.49 & \\
\hline Mamá me llama tonto & 196 & 0 & 6 & 1.15 & 1.76 & \\
\hline Mamá me dice me correrá a golpes & 196 & 0 & 6 & 0.20 & 0.93 & \\
\hline Mamá me pega en la cara & 196 & 0 & 6 & 0.24 & 0.98 & \\
\hline VIOLENCIA ENTRE PADRES & & & & & & .91 \\
\hline Papá insulta con malas palabras a mamá & 203 & 0 & 6 & 1.01 & 1.77 & \\
\hline Mamá insulta con malas palabras a papá & 203 & 0 & 6 & 0.82 & 1.59 & \\
\hline Papá le tira con cosas a mamá & 203 & 0 & 6 & 0.40 & 1.20 & \\
\hline Papá empuja o agarra violentamente a mamá & 203 & 0 & 6 & 0.38 & 1.20 & \\
\hline Mamá empuja o agarra violentamente a papá & 203 & 0 & 6 & 0.27 & 1.00 & \\
\hline Papá le pega una cachetada a mamá & 203 & 0 & 6 & 0.18 & 0.77 & \\
\hline Mamá le pega una cachetada a papá & 203 & 0 & 6 & 0.23 & 0.85 & \\
\hline Papá le pegó una patada, puñetazo a mamá & 203 & 0 & 6 & 0.26 & 1.02 & \\
\hline Mamá le pegó una patada, puñetazo a papá & 203 & 0 & 6 & 0.19 & 0.81 & \\
\hline Papá golpear a mamá durante algunos minutos & 203 & 0 & 6 & 0.26 & 1.03 & \\
\hline Papá le ha roto la ropa o destruido cosas a mamá & 203 & 0 & 6 & 0.18 & 0.79 & \\
\hline Mamá le ha roto la ropa o destruido cosas a papá & 203 & 0 & 6 & 0.24 & 0.97 & \\
\hline ALCOHOL DE LA MADRE & & & & & & .60 \\
\hline Veces que bebe alcohol los fines de semana & 203 & 0 & 9 & 0.43 & 0.94 & \\
\hline Veces que bebe alcohol entre semana & 203 & 0 & 5 & 0.24 & 0.70 & \\
\hline Cantidad que bebe en cada ocasión & 203 & 0 & 6 & 0.49 & 0.94 & \\
\hline ESCALA y Variables & $\mathrm{N}$ & Min & Max & Media & D.E. & alfa \\
\hline CONDUCTA ESCOLAR & & & & & & .82 \\
\hline Te mandaron castigado con el director & 202 & 0 & 5 & 0.44 & 1.09 & \\
\hline Faltaste a la escuela & 202 & 0 & 5 & 1.26 & 1.25 & \\
\hline No te juntaste con alguien en la escuela & 202 & 0 & 5 & 0.96 & 1.58 & \\
\hline Participaste en actividades de tu escuela & 202 & 0 & 5 & 1.69 & 1.78 & \\
\hline Te suspendieron de la escuela & 202 & 0 & 5 & 0.42 & 1.11 & \\
\hline Dejaste de ir a la escuela & 202 & 0 & 5 & 0.40 & 1.10 & \\
\hline Te expulsaron de la escuela & 202 & 0 & 5 & 0.44 & 1.16 & \\
\hline Te cambiaron de escuela & 202 & 0 & 5 & 0.43 & 1.07 & \\
\hline Respondiste en clase en voz alta sin ser tu turno & 202 & 0 & 5 & 0.72 & 1.30 & \\
\hline Te costó trabajo desobedecer las reglas & 202 & 0 & 5 & 1.04 & 1.53 & \\
\hline Discutes con tus compañeros & 202 & 0 & 5 & 1.18 & 1.54 & \\
\hline AMBIENTE CASA & & & & & & .60 \\
\hline Caliente & 198 & 0 & 10 & 4.92 & 3.14 & \\
\hline Insegura & 198 & 0 & 10 & 2.84 & 3.33 & \\
\hline Ruidosa & 198 & 0 & 10 & 3.35 & 3.33 & \\
\hline Deteriorada & 198 & 0 & 10 & 2.19 & 2.88 & \\
\hline
\end{tabular}


Tabla 2

Continuación

\begin{tabular}{|c|c|c|c|c|c|c|}
\hline ESCALA y Variables & $\mathrm{N}$ & Min & Max & Media & D.E. & alfa \\
\hline AMBIENTE COLONIA & & & & & & .80 \\
\hline Expendios de venta de cerveza & 197 & 0 & 10 & 2.45 & 3.01 & \\
\hline Pleitos barrio & 197 & 0 & 10 & 2.42 & 7.84 & \\
\hline Peligroso barrio colonia & 197 & 0 & 10 & 3.28 & 2.89 & \\
\hline Ruidosa & 197 & 0 & 10 & 3.85 & 3.12 & \\
\hline Venta de drogas & 197 & 0 & 10 & 3.08 & 3.33 & \\
\hline Sucia colonia & 197 & 0 & 10 & 3.10 & 2.82 & \\
\hline Oscura colonia & 197 & 0 & 10 & 3.63 & 3.13 & \\
\hline Vagos & 197 & 0 & 10 & 4.27 & 3.27 & \\
\hline Borracho & 197 & 0 & 10 & 3.35 & 3.09 & \\
\hline Descuidada & 197 & 0 & 10 & 3.40 & 2.96 & \\
\hline Hay gente muchos lugares & 197 & 0 & 10 & 3.91 & 3.23 & \\
\hline AMBIENTE ESCOLAR & & & & & & .78 \\
\hline Hay pandillas en escuela & 197 & 0 & 6 & 0.31 & 0.91 & \\
\hline Qué tanta droga se vende en la escuela & 197 & 0 & 10 & 1.00 & 2.79 & \\
\hline Qué tan peligrosa es la escuela & 197 & 0 & 10 & 2.71 & 2.82 & \\
\hline Qué tan sucia está la escuela & 197 & 0 & 10 & 1.68 & 2.37 & \\
\hline Qué tantos vagos hay en escuela & 197 & 0 & 10 & 2.71 & 3.08 & \\
\hline Qué tan descuidada está la escuela & 197 & 0 & 10 & 2.15 & 2.84 & \\
\hline CREENCIAS Y ACTITUDES ANTISOCIALES & & & & & & .82 \\
\hline Para progresar: hacer cosas no buenas & 196 & 0 & 6 & 1.19 & 1.56 & \\
\hline Hacer trampas y mentir no es malo & 196 & 0 & 6 & 1.95 & 1.68 & \\
\hline Cuando no sigo reglas me siento culpable & 196 & 0 & 6 & 2.06 & 1.62 & \\
\hline Trato de obtener las cosas que quiero, como sea & 196 & 0 & 6 & 1.22 & 1.51 & \\
\hline Hago lo que me causa placer, aquí y ahora & 196 & 0 & 6 & 1.12 & 1.53 & \\
\hline La gente que viola las leyes siempre es atrapada & 196 & 0 & 6 & 1.71 & 1.69 & \\
\hline No dedico esfuerzo en prepararme para futuro & 196 & 0 & 6 & 1.08 & 1.54 & \\
\hline Algunas veces tomo riesgos sólo por placer & 196 & 0 & 6 & 1.26 & 1.50 & \\
\hline Me pruebo haciendo cosas arriesgadas & 196 & 0 & 6 & 1.30 & 1.54 & \\
\hline CONDUCTA ANTISOCIAL & & & & & & .88 \\
\hline Robaste cosas de almacenes estando abiertos & 195 & 0 & 6 & 0.33 & 1.11 & \\
\hline Conseguiste dinero amenazando a personas & 195 & 0 & 6 & 0.20 & 0.88 & \\
\hline Perteneciste a alguna pandilla & 195 & 0 & 1 & 0.04 & 0.21 & \\
\hline Veces que te metiste en peleas o disturbios & 195 & 0 & 6 & 0.31 & 1.06 & \\
\hline Veces que tomaste licor o drogas & 195 & 0 & 8 & 0.40 & 1.40 & \\
\hline Veces que robaste & 195 & 0 & 9 & 0.18 & 0.90 & \\
\hline Veces que cargaste un arma blanca o de fuego & 195 & 0 & 9 & 0.34 & 1.28 & \\
\hline Veces que heriste a personas con armas & 195 & 0 & 7 & 0.23 & 1.00 & \\
\hline
\end{tabular}

escuelas, y las que existen se encuentran en malas condiciones), se presenta venta de drogas y licor y esto pudiera originar un ambiente de descuido, propicio para la violencia (Abrahamson, 1996; Bursik, 2001).

Los resultados de nuestro estudio también mostraron el efecto positivo de un ambiente familiar inadecuado en la conducta antisocial de los menores. Tal como lo menciona Bronfenbrenner (1987), la familia puede funcionar como un contexto efectivo de desarrollo del niño. Sin embargo, los conflictos familiares, la pérdida de los padres, y la falta de habilidades de crianza son los factores que pudieran intervenir en el desarrollo de la delincuencia o de la conducta antisocial en los menores (Juby \& Farrington, 2001; Wells \& Rankin, 1991). La delincuencia juvenil según algunos autores, es una de las consecuencias negativas predecibles de condiciones familiares inadecuadas como ocurre en el caso de la violencia familiar. Algunos estudios indican que, los niños/as que son testigos de la violencia o que reciben el maltrato directamente pudieran desarrollar conducta antisocial o delictiva (McCord, 2001; Morrison \& Cherlin, 1995; Widom \& Ames, 1994).

Nuestros datos parecen mostrar una visión más completa del desarrollo de la delincuencia juvenil, en comparación 


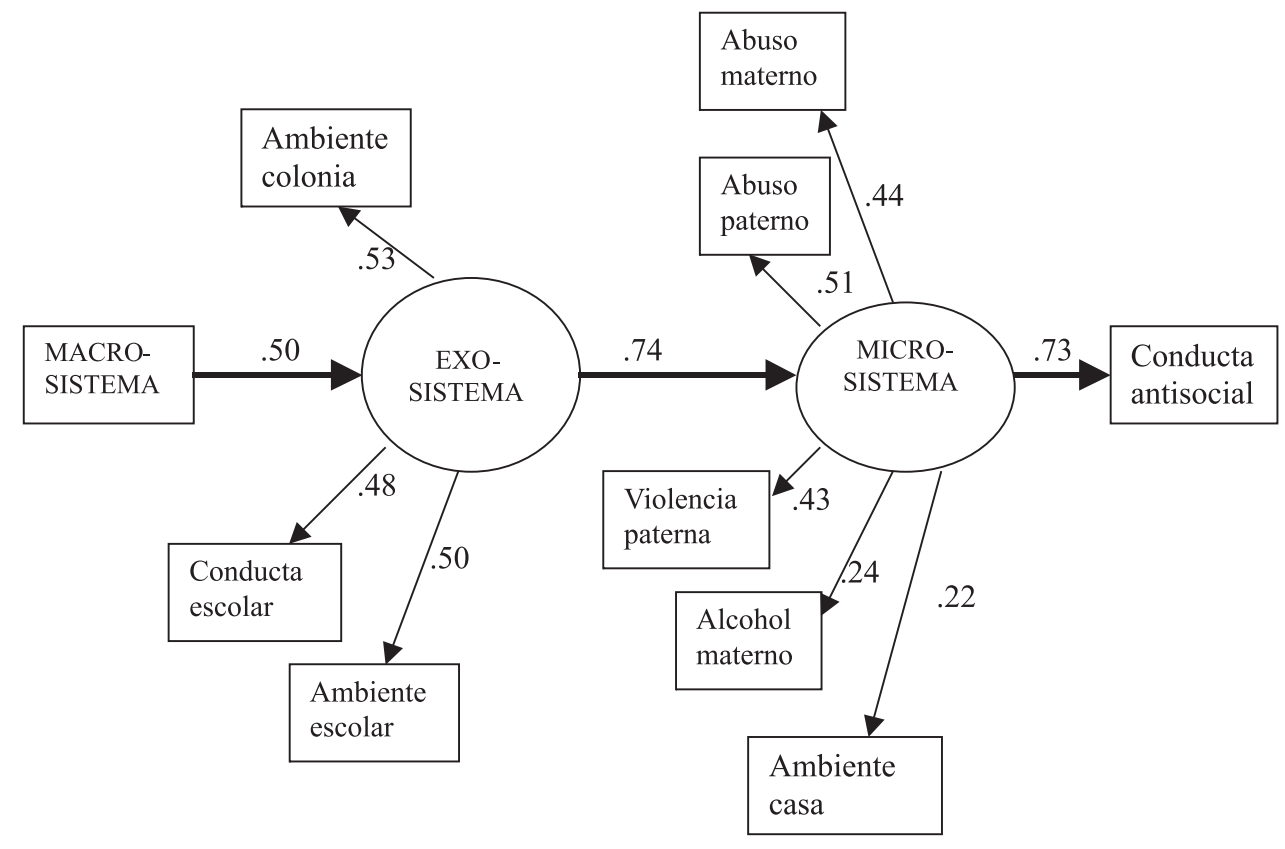

Figura 1. Modelo ecológico de predictores de la conducta antisocial. Todos los pesos factoriales y los coeficientes estructurales son significativos $(p<.05)$. Bondad de ajuste: $\chi^{2}=53.39$ (30 g.l. $), p=.005 ;$ IANN=.90; IAC=.93; $R M S E A=.04 ; R^{2}=.56$.

con los derivados de esquemas más específicos (marcos explicativos no ecológicos). Estos datos indicarían que la delincuencia o la conducta antisocial juvenil son fenómenos que reciben influencias no sólo multifactoriales sino multicontextuales también. La teoría ecológica presenta una alternativa para modelar los efectos de estos factores. De la misma manera este modelo teórico nos proporciona una pauta para la propuesta de sistemas de intervención. Siguiendo este esquema se propone que los programas de intervención consideren mecanismos que impacten en todos los contextos, si se esperan resultados efectivos para enfrentar los problemas abordados. Las intervenciones a nivel individual no serán efectivas si no se toman en cuenta todos los escenarios en los que se desarrollan los jóvenes en nuestras sociedades.

\section{Referencias}

Abrahamson, M. (1996). Urban enclaves: Identity and place in America. Nueva York: St. Martin's Press.

American Psychological Association's Comission on Youth Violence (1993). Violence and youth: Psychology's response. Washington, DC: Autor.

Angenent, H., \& Man, A. (1996). Background factors of juvenile delinquency. Nueva York: Peter Lang Publishing.

Belsky, J. (1980). Child maltreatment: An ecological integration. American Psychologist, 35, 320-335.

Bentler, P. M. (1995). EQS. Structural Equations Program Manual. Encino, California: Multivariate Software.

Brook, J., \& Newcomb, M. (1995). Childhood aggression and unconventionality: Impact on later academic achievement, drug use, and workforce involvement. Journal of Genetic Psychology, 156, 139-410.

Browne, M. W., \& Cudeck, R. (1993). Alternative ways of assessing model fit. In K. A. Bollen \& J. S. Long (Orgs.), Testing structural equation models (pp. 136-162). Thousand Oaks, California: Sage.
Bronfenbrenner, U. (1987). La ecología del desarrollo humano. Barcelona, España: Paidós.

Bronfenbrenner, U., \& Ceci, S. J. (1994). Nature-nurture reconceptualized: A bioecological model. Psychological Review, 101(4), 568-586.

Bursik, R. J. (2001). Community. In W. Kluver (Org.), Handbook of youth and justice (pp. 265-275). Nueva York: Academic/Plenum.

Corral-Verdugo, V. (2002). Structural equation modeling. In R.B. Bechtel \& A. Churchman(Orgs.), Handbookof Environmental Psychology(PP; 256-270). Nueva York: Wiley.

Emery, R., \& Laumann-Billings, L. (1998). An overview of the nature, causes, and consequences of abusive family relationships. American Psychologist, 53, 121-135.

Freud, S. (1930). Civilization and its discontents. Londres: Hogarth.

Frías, M., Corral, V., Moreno, C., \& Rodríguez, I. (2000). Predictores de la delincuencia juvenil. La Psicología Social en México, 8, 479-485.

Frías, M., Ramírez, J., Soto, R., Castell, I. \& Corral, V. (2000). Repercusiones del maltrato infantil: un estudio con niños en alto riesgo de maltrato. $L a$ Psicología Social en México, 8, 486-492.

Gaustad, J. (1992). Schools attack the roots of violence. ERIC Digest No. 63. Eugene, Oregon: Clearinghouse on Educational Management.

Gobierno del Estado de Sonora (1998). Anuario estadístico del Estado de Sonora. Hermosillo, Sonora: Autor.

Hagan, J., Hefler, G., Classen, G., Boehnke, K., \& Merkens, H. (1998). Subterranean sources of subcultural delinquency beyond the American dream. Criminology, 36, 309-342.

Instituto Nacional de Estadística, Geografía e Informática. (1998). Anuario estadístico del Estado de Sonora. México: Autor.

Juby , H., \& Farrington, D. P. (2001). Disentangling the link between disrupted families and delinquency. British Journal of Criminology, 41, 22-40.

Lerner, R. M., \& Galambos, N. L. (1998). Adolescent development: challenges and opportunities for research, programs, and policies. Annual Review of Psychology, 49, 413-446.

Loeber, R., \& Hay, D. (1997). Key issues in the development of aggression and violence from childhood to early adulthood. Annual Review of Psychology, 48, 371-410. 
Loeber, R., Keenan, K., \& Zhang, Q. (1997). Boys' experimentation and persistence in developmental pathways toward serious delinquency. Journal of Child and Family Studies, 6, 321-357.

Lorenz, K. (1966). On aggression. Nueva York: Harcourt, Brace \& World.

Lotz, R., \& Lee, L. (1999). Sociability, school experience, and delinquency. Youth \& Society, 31, 199-223.

McCord, J. (2001). Forging criminals in the family. In W. Kluber (Org.), Handbook of youth and justice (pp. 223-235). Nueva York: Academic/Plenum.

McCord, J. (1996). Family as crucible for violence. Journal of Family Psychology, 10, 147-152.

Morrison, D. R., \& Cherlin, A. (1995). The divorce process and young children's well-being: a prospective analysis. Journal of Marriage and Family, 57, 800-812.

National Research Council. (1993). Understanding and preventing violence. Washington, DC: National Academic Press.

Nunally, J. C., \& Bernstein, I. H. (1994). Psychometric theory. Nueva York: McGraw-Hill.

Ollendick, T. H. (1996). Violence in youth: where do we go from here? Behavior therapy’s response. Behavior Therapy, 27, 485-514.

Peterson, R. D., Krivo, L. J., Harris, M. A. (2000). Disadvantage and neighborhood violent crime: do local institutions matter? Journal of Research in Crime an Delinquency, 37(1), 31-63.

Peterson, R. D., \& Krivo, L. J. (1993). Racial segregation and black urban homicide. Social Forces, 71, 1001-1026.

Reich, W., \& Herjanic, B. (1989). The diagnostic interview for children and adolescents. Manuscrito no publicado. Department of Psychiatry, Washington University, St. Louis, Missouri.

Sampson, R. J. (1997). The embeddedness of child and adolescent development: a community-level perspective on urban violence. In J. McCord (Org.),
Violence and childhood in the inner city (pp. 40-67). Cambridge: Cambridge University Press.

Sampson, R. J., Raudenbush, S. W., \& Earls, F. (1997). Neighborhoods and violent crime: A multilevel study of collective efficacy. Science, 277, 918924.

Straus, M. A. (1979). Measuring intrafamily conflict and violence. The Conflict Scale (CTS). Journal of Marriage and the Family, 41, 75-88.

Straus, M. A., Gelles, R., \& Steinmetz, H. (1980). Behind closed doors: violence in the American family. Garden City, Nueva York: Anchor.

Straus, M. A. (1990). The Conflicts Tactics Scale and its critics: an evaluation an new data on validity and reliability. In M. A. Strauss \& R. J. Gelles (Orgs.), Physical violence in American families (pp. 49-73). New Brunswick, New Jersey: Transaction Publishers.

Straus, M. (1991). Discipline and deviance: physical punishment of children and violence in adulthood. Durham: University of New Hampshire, Family Research Laboratory.

Straus, M. A., Sugarman, D. B., \& Giles-Sims, J. (1997). Spanking parents and subsequent antisocial behavior of children. Archives of Pediatric Adolescent Medicine, 151, 761-767.

Taylor, R. B. (1997). Social order \& disorder of street blocks and neighborhoods: ecology, microecology, and the systemic model of social disorganization. Journal of Research in Crime and Delinquency, 34, 133-155.

Vazsonyi, A., \& Flannery, D. (1997). Early adolescents delinquent behavior: associations with family and school domains. Journal of Early Adolescence, 17(3), 271-293.

Wells, L. E., \& Rankin, J. H. (1991). Families and delinquency: a meta-analysis of the impact of broken homes. Social Problems, 38, 71-93.

Widom, C. S. \& Ames, A. (1994). Criminal consequences of childhood sexual victimization. Child Abuse \& Neglect, 18, 303-318.

\section{Nota}

1 Esta investigación se desarrolló gracias al apoyo del Consejo Nacional de Ciencia Y Tecnología (CONACYT) de México (Proyecto 35166-H).

Martha Frías-Armenta, Doutora em Psicologia pela University of Arizona (Tucson, EUA), é Professora Investigadora no Departamento de Direito da Universidad de Sonora, Hermosillo, México.

Amelia Eréndida López-Escobar e Sylvia Guadalupe Díaz-Méndez são Licenciadas en Psicología da Universidad de Sonora, México.

Endereço para correspondência: Martha Frías Armenta, Sevilla 6, Fracc. Casa Grande - Sección III, Hermosillo, Sonora, 83240, México. E-mail: marthafrias@sociales.uson.mx. 